\title{
The Expression Pattern of Prostaglandin-Endoperoxide Synthase-2 in Immature Oocytes and Surrounding Cumulus Cells May Explain A Disrupted Oocyte Maturation Process
}

\author{
Goktan KUSPINAR', Berrin AVCI',2
}

Bursa, Turkey

\begin{abstract}
OBJECTIVE: To evaluate the correlation between the gene expression of prostaglandin-endoperoxide synthase-2 in the cumulus-oocyte complex with the level of prostaglandin-endoperoxide synthase-2 in follicular fluid and follicle diameter, oocyte maturation, and fertilization capacity.

STUDY DESIGN: As the study group, 26 cumulus-oocyte complexes and 26 follicular fluids obtained from immature $(n=10)$ or unfertilized mature oocytes $(n=16)$ and as the control group, 26 cumulus complexes and 26 follicular fluids surrounding mature and fertilized oocytes were retrieved one by one totally from 32 patients in 32 intracytoplasmic sperm injection cycles.

RESULTS: There was no significant efficacy of prostaglandin-endoperoxide synthase-2 gene expressions in cumulus complexes and the level of prostaglandin-endoperoxide synthase- 2 in follicular fluids in terms of oocyte maturation stage. The level of prostaglandin-endoperoxide synthase-2 in follicular fluids and follicle diameters showed a significantly positive correlation in the mature and fertilized oocyte group $(r=0.414 ; p=0.035)$.

CONCLUSIONS: Although the prostaglandin-endoperoxide synthase-2 gene expressions in immature oocytes and their cumulus cells were similar to those in oocytes that have completed their nuclear and cytoplasmic maturation, the level of prostaglandin-endoperoxide synthases- 2 in the follicular fluid and follicle diameter correlation may lead to new clinical approaches in cases of premature follicular rupture before oocyte maturation is completed.
\end{abstract}

Keywords: Follicle micro-environment, Oocyte maturation, Prostaglandin-endoperoxide synthase-2

Gynecol Obstet Reprod Med 2021;27(3):242-247

\section{Introduction}

Prostaglandins (PGs) perform a variety of roles in female reproductive functions including ovulation, fertilization and implantation (1). The prostaglandin-endoperoxide synthase (PTGS), PTGS-1 and PTGS-2 isoenzymes are the primary en-

${ }^{1}$ Department of Histology and Embryology, Bursa Uludag University School of Medicine, Bursa, Turkey.

2 Department of Gynecology and Obstetrics ART Center, Bursa Uludag University School of Medicine, Bursa, Turkey.

Address of Correspondence: Berrin Avci

Department of Histology and Embryology

Bursa Uludag University School of

Medicine, 16059 Gorukle, Bursa, Turkey

berrin@uludag.edu.tr

Submitted for Publication: 13.02.2020 Revised for Publication 12.04.2020

Accepted for Publication: 23.04.2020 Online Published: 30.04.2020

ORCID IDs of the authors: GK: 0000-0002-0338-8368

BA: 0000-0001-8135-5468

\begin{tabular}{|c|c|}
\hline \multirow{3}{*}{ 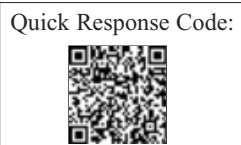 } & Access this article online \\
\hline & $\begin{array}{l}\text { Website: www.gorm.com.tr } \\
\text { e- mail: info@gorm.com.tr }\end{array}$ \\
\hline & DOI:10.21613/GORM.2020.1069 \\
\hline
\end{tabular}

How to cite this article: Kuspinar G. Avci B. The Expression Pattern of Prostaglandin-Endoperoxide Synthase-2 in Immature Oocytes and Surrounding Cumulus Cells May Explain A Disrupted Oocyte Maturation Process. Gynecol Obstet Reprod Med 2021;27(3):242-247 zymes responsible for the production of PGs. While PTGS-1 is a constitutive form of the enzyme, PTGS-2 can be induced by inflammatory stimulants, cytokines, and growth hormones (2). Pre-ovulatory bovine follicular granulosa cells and cumulus cells (CCs) have shown PTGS-2 gene expression, although PTGS-1 expression has not been observed in the in vitro maturation process of isolated cumulus-oocyte complex (COCs) (3). PTGS-2 -/- deficient female mice have been reported as infertile due to ovulation, fertilization, and implantation defects (4). In the murine ovulation model, PTGS-2 plays an important enzymatic role in CC expansion and rupture of the follicle (5-8). In the process of ovulation, the application of PTGS-2 inhibitor in a monkey model caused a poor fertilization rate of mature oocytes, revealing that accurate timing of PTGS-2 expression and activity is necessary for fertilization capacity even after oocyte nuclear maturation (9).

In assisted reproduction technology (ART) cycles, controlled ovarian hyperstimulation is used for follicular recruitment. Follicular size and serum estradiol concentration are commonly used as indicators of oocyte maturity (10). Oocytes from follicles with mean diameters greater than $12 \mathrm{~mm}$ have been reported to have significantly higher fertilization and cleavage rates (11). A positive correlation has also been found 
between follicular size and the presence of mature oocytes (12). The measurement of follicular size before retrieval might be an indicator of the fertilization potential of oocytes in a cycle (13). However, in intracytoplasmic sperm injection (ICSI) cycles, the data are still scanty regarding follicular size and fertilization rate.

The aim of the study was thus two-fold: first, to explain the disrupted oocyte maturation process and fertilization defects with the gene expression of PTGS-2 in oocytes and surrounding cumulus cells, and second, to analyze whether follicular diameter has a relation with the level of follicular fluid PTGS2 in follicles that contain mature or immature oocytes.

\section{Material and method}

This clinical trial was conducted between September 2015 and May 2016 with patients who participated in the Assisted Reproduction Program of the University Hospital and underwent ovarian stimulation for ICSI cycles. The patient group allocation was performed according to the retrieved oocyte maturation stage (Figure I). Patient exclusion criteria were: documented endometriosis and female age of $>39$ years old due to the detrimental effects on oocyte quality (14). Patients with male factor such as oligoasthenoteratozoospermia (OAT) and azoospermia were excluded to avoid possible effects on oocyte fertilization status. Approval for the study was granted by the local ethics committee (no: 2015-17/14) and informed consent was obtained from each participant.

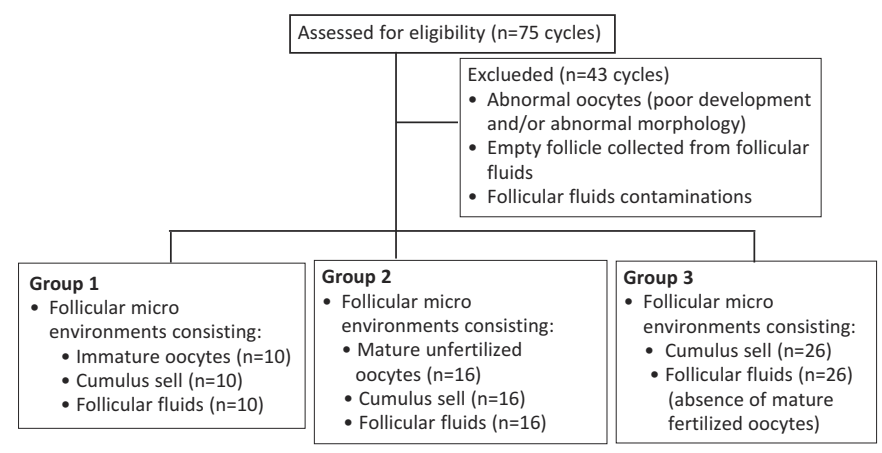

Figure 1: Sample allocation diagram

Patients were stimulated with 150-375 IU per day of recombinant FSH (rFSH) (Gonal F, Merck-Serono, GERMANY) that was started on day 2 of the menstrual cycle. Pituitary suppression was achieved with $\mathrm{GnRH}$ antagonists (Cetrotide, Merck-Serono, Germany) during flexible antagonist protocol. Serum estradiol (E2) levels and follicle growth were monitored daily. When at least two or more follicles reached $\geq 17 \mathrm{~mm}$ diameter, $250 \mu \mathrm{g} / 0,5 \mathrm{ml}$ recombinant $\beta$-hCG (Ovitrelle, Merck-Serono, Germany) was administered (14). The rate of response to $\mathrm{COH}$ was calculated as: [(retrieved oocyte number / AFC) x 100]. Additionally, the $\mathrm{COH}$ response and total recombinant FSH dose relation were evaluated due to avoiding the possible effect of recombinant FSH both on follicle diameter and oocyte maturation or fertilization.
Oocyte pick-up (OPU) was performed 34-36 hours after ovulation trigger, by vaginal puncture with a $17 \mathrm{~mm}$ gauge needle with ultrasound guidance. Before aspiration, the follicle diameter was calculated with transvaginal ultrasound and the largest follicle in each ovary was aspirated firstly. The aspirated follicular fluids (FFs) and cumulus-oocyte complexes (COCs) were kept apart from others in order to prevent contamination of other follicle micro-environments. The first FFs and COCs aspirated from each ovary of the patient were included in the study. Aspiration of the other follicles was performed in accordance with the routine application. After aspiration, COCs were isolated from FFs. COCs were washed in GMOPs medium (Vitrolife, Goteborg, Sweden) and immediately incubated in fertilization medium (Vitrolife, Goteborg, Sweden) in marked four-well dishes at $37{ }^{\circ} \mathrm{C}$ in a humidified atmosphere of $6 \% \mathrm{CO}_{2}, 5 \% \mathrm{O}_{2}$ (the balance gas was nitrogen) using Hera-Cell incubators (Thermo Fisher Scientific Inc., Massachusetts, USA). After 2-4 hours, CCs were removed form oocytes firstly mechanically with a ppd injector to retrieve cumulus cells. Then oocytes were denuded with enzymatic treatment with $80 \mathrm{IU} / \mathrm{mL}$ hyaluronidase solution to prepare for ICSI. Afterward, the samples were placed in a cleavage medium (Vitrolife, Goteborg, Sweden).

FF samples were centrifuged at $1.600 \mathrm{~g}$ for 3 minutes and separated from other cells and components. The supernatant was obtained after centrifugation and stored at $-80{ }^{\circ} \mathrm{C}$ until ELISA was performed.

After denudation, the oocytes were evaluated for nuclear maturity and classified as germinal vesicle (GV), MetaphaseI (MI) or Metaphase-II (MII) under inverted microscopy. The evaluation was based on the presence of the GV, GV breakdown or extrusion of the first polar body, respectively. ICSI was performed with oocytes at the M-II stage and then oocytes were incubated for pre-embryological development. The study groups are shown in figure 1.

Total RNA was extracted from the oocyte and CCs using the Quick-RNA TM MicroPrep Kit (Zymo Research Corp., USA) according to the manufacturer's protocol. Complementary DNAs (cDNAs) were synthesized using the SensiFAST cDNA Synthesis Kit (Bioline Life Science Corp., USA). All cDNA was frozen at $-80^{\circ} \mathrm{C}$ until it was used for RT-PCR. The sequence of the primer pairs used for PCR was: (i) PTGS-2 forward 5'-GTTCCACCCGCAGTACAGAA-3' and (ii) reverse 5'-AGGGCTTCAGCATAAAGCGT -3', giving a PCR product of 106 base pairs.

The genes coding glyceraldehyde-3-phosphate-dehydrogenase (GAPDH) were selected as reference genes for normalizing the PCR data. The sequences of the forward and reverse primers used for PCR were: forward 5'GAGTCAACGGATTTGGTCGT-3' and reverse 5'GACAAGCTTCCCGTTCTCAG-3', giving a PCR product of 185 base pairs. 
Real-time quantitative polymerase chain reaction (RTPCR) was used to quantify the expression of the test genes in the oocyte and cumulus cells from the same follicles. The relative quantification of gene expression was calculated using the $2^{-\Delta \Delta C T}$ method. RT-PCR was performed on the Light Cycler $^{\circledR} 480$ Real-Time PCR System (Roche Ltd., Switzerland) using the SensiFAST ${ }^{\text {TM }}$ SYBR $^{\circledR}$ Hi-ROX Kit (Bioline Life Science Comp., USA). Each assay was performed in triplicate and three negative controls were run for each assay: no template, no reverse transcriptase, and no RNA in the reverse transcriptase reaction.

FF PTGS-2 levels were measured using commercial enzyme-linked immunosorbent assay (ELISA) kits according to the manufacturer's instructions (Sunred Biological Technology, Shangai). The PTGS-2 assay had a sensitivity of $2,125 \mathrm{U} / \mathrm{L}$ detection range of 3 to $1.000 \mathrm{U} / \mathrm{L}$ and intra-assay $\mathrm{CV}$ of $<8 \%$. Serial dilutions of goose samples with high readings were used to generate binding patterns parallel to the standard curves, to validate the assays for measuring goose samples.

The non-normally distributed variables were defined with the median value (minimum-maximum value). The MannWhitney U (for comparison of ranks between two groups) or the Kruskal-Wallis (to compare more than two groups) nonparametric tests for variables were used in the statistical analysis. For analyses of the correlations of non-normally distributed variables, the Spearman Rho test was performed. Linear regression analysis (enter method) was performed for identifying factors associated with ovarian response to COS between baseline clinical characteristics of patients. A twosided $\mathrm{p}$-value $<0.05$ was considered statistically significant. These procedures were undertaken using SPSS 23.0 (IBM) software.

\section{Results}

Patient age, body mass index (BMI), basal antral follicle counts, basal endocrine parameters, estradiol levels on the day of ovulation trigger, and total gonadotrophin doses (baseline clinical characteristics of patients) were compared among patients comprising the study groups and these are shown in table I. The overall mean age at the time of the study was 31.87 years (range, $24-39$ years). The mean BMI index value was 25.87 (16.30-37.40). The mean serum Anti-Müllerian Hormone (AMH) level was $2.39(0.01-8.4) \mathrm{ng} / \mathrm{dL}$ and the mean antral follicle count (AFC) number was 8.73 (1-16) with no statistically significant difference between the groups. The rate of response to $\mathrm{COH}$ was compared among patients comprising the study groups and this showed no differences $(p=0.09)$. In the multiple linear regression analyses, the $\mathrm{COH}$ response was not associated with patient age $(\beta=0.762$, $p=0.460)$, BMI $(\beta=-0.62, p=0.700)$, or the total gonadotrophin dose $(\beta=0.32, p=0.06)$ for all patients. The rate of $\mathrm{COH}$ response was only associated with patient AMH levels ( $\beta=0.671, p=0.01$ ) for all patients, although the mean AMH levels and total gonadotrophin doses were similar between the groups ( $p=0.867 ; p=0.430$ respectively).

The relative expression levels of PTGS-2 were evaluated in the CCs according to the nuclear maturation stage of oocytes and fertilized versus unfertilized oocytes. In terms of the nuclear maturation stage; PTGS-2 expression in immature oocyte CCs $(n=10)$ tended to increase 1.67-fold higher than in mature oocyte CCs $(n=42)$, although there was no significant difference (95\% CI 0.00001 to $1.53 ; p=0.443$ ) (Figure 2). In terms of the cytoplasmic maturation stage (referred fertilization ability); PTGS-2 expression in the unfertilized oocyte CCs $(n=16)$ tended to increase 2.82-fold higher than in fertilized oocyte CCs $(\mathrm{n}=26)$, with no significant difference $(95 \%$ CI 0.00001 to $6.64 ; p=0.057$ ) (Figure 2).

Table I: Baseline clinical characteristics of patients in total and in groups

\begin{tabular}{|c|c|c|c|c|c|}
\hline & Overall & Group 1 & Group 2 & Group 3 & $p$ \\
\hline Age & $31.87(24-39)$ & $31(27-34)$ & $33(24-39)$ & $34(25-39)$ & 0.788 \\
\hline Body Mass Index & $25.87(16.30-37.40)$ & $31(1937.40)$ & $25(21.50-31.50)$ & $26.4(16.3-34)$ & 0.404 \\
\hline Anti-mullerian & $2.39(0.01-8.4)$ & $1.43(0.1-8)$ & $2.3(0.01-7.40)$ & $1.57(0.1-8.40)$ & 0.867 \\
\hline \multicolumn{6}{|l|}{ Hormone (ng/dL } \\
\hline Antral follicle count (AFC) & $8.73(1-16)$ & $6(1-16)$ & $8(1-15)$ & $9(2-12)$ & 0.673 \\
\hline Follicle stimulating & $5.63(3.00-10.5)$ & $6.5(3-10.5)$ & $4.9(3-9.8)$ & $6.5(4.20-8.60)$ & 0.823 \\
\hline \multicolumn{6}{|l|}{ Hormone (mLU/mL) } \\
\hline Luteinizing hormone (mLU/mL) & $5.05(1.60-11.30)$ & $3.7(1.5-10.1)$ & $4.3(2.5-6.1)$ & $4.8(1.6-11.3)$ & 0.671 \\
\hline Estradiol (ng/mL) & $52.1(10-201)$ & $49(20.1-200)$ & $33(10-201)$ & $56(14-121)$ & 0.280 \\
\hline Estradiol on trigger day $(\mathrm{ng} / \mathrm{mL})$ & $1394.87(86-5000)$ & $230(86-1591)$ & $877(86-5000)$ & $1273(314-4361)$ & 0.124 \\
\hline Total gonadotropin dose (Units) & $2400(750-4200)$ & $2100(750-3600)$ & $2250(1600-3600)$ & $2437.5(900-4200)$ & 0.430 \\
\hline Success rate of controlled & 1.1 & $0.67^{x}$ & $1.22^{y}$ & $1.27^{y}$ & $0.09^{*}$ \\
\hline
\end{tabular}

*The Kruskal-Wallis test for comparison of ranks between groups. Data are given as median (min-max) 


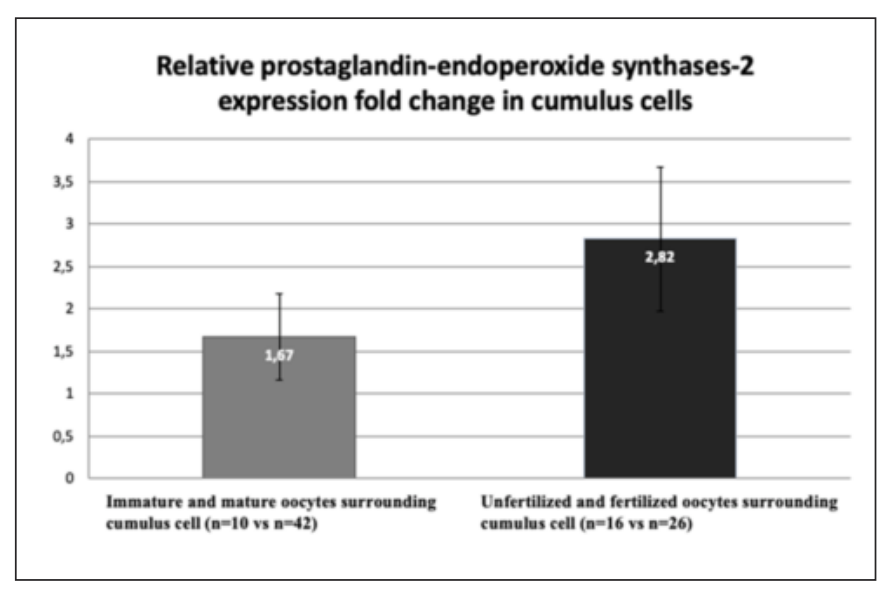

Figure 2: The prostaglandin-endoperoxide synthase-2 (PTGS2) expression in immature oocytes surrounding CCs $(n=10)$ increased 1.67-fold higher than in mature oocytes surrounding CCs ( $n=42)$ although there were no significant differences (95\% Cl 0.00001 to 1.53; $p=0.443$ ). In terms of the cytoplasmic maturation stage (referred fertilization ability) PTGS-2 expression in unfertilized oocytes surrounding CCs $(n=16)$ increased 2.82-fold higher than in fertilized oocytes surrounding CCs $(n=26)$ although there were no significant differences $(95 \% \mathrm{Cl}$ 0.00001 to $6.64 ; p=0.057$ ). All data were normalized by using the $2^{-\triangle \Delta C T}$ method.

The relative expression level of PTGS-2 in oocytes was compared according to the oocyte maturation stage. In immature oocytes, PTGS-2 expression tended to decrease 1.35-fold lower than in unfertilized mature oocytes, with no significant difference ( $95 \%$ CI 0.00001 to $1.48 ; p=0.174$ ). Mature and fertilized oocytes were not included in this analysis as there could have been a potential for embryo development.

Measurement for the diameter of the first aspirated follicles was made with transvaginal ultrasound, before follicle aspiration (Table II). The mean follicular diameter was measured as 18 (14-20) $\mathrm{mm}$ in Group 1, 20.6 (17-24.5) $\mathrm{mm}$ in Group 2 and $19.65(17-22.5) \mathrm{mm}$ in Group 3. A statistically significant difference was determined between Groups 1 and 2 $(p=0.002)$ and between Groups 1 and $3(p=0.012)$. The levels of FF PTGS-2 were evaluated between the groups (Table II). The mean FF PTGS-2 concentrations were 162.18 (126.17291.58), 156.51 (138.31-626.88), and 163.63 (83.96-484.83) $\mathrm{u} / \mathrm{L}$ respectively in Groups 1, 2 and 3, and the results were comparable between the groups $(p=0.504)$. The correlations between follicle diameter and FF PTGS-2 levels were evaluated between the groups. With higher FF PTGS-2 levels only in Group 3, a statistically significant positive correlation was determined between follicular diameter and FF PTGS-2 levels $(r=0.414 ; p=0.035)$ (Figure 3).

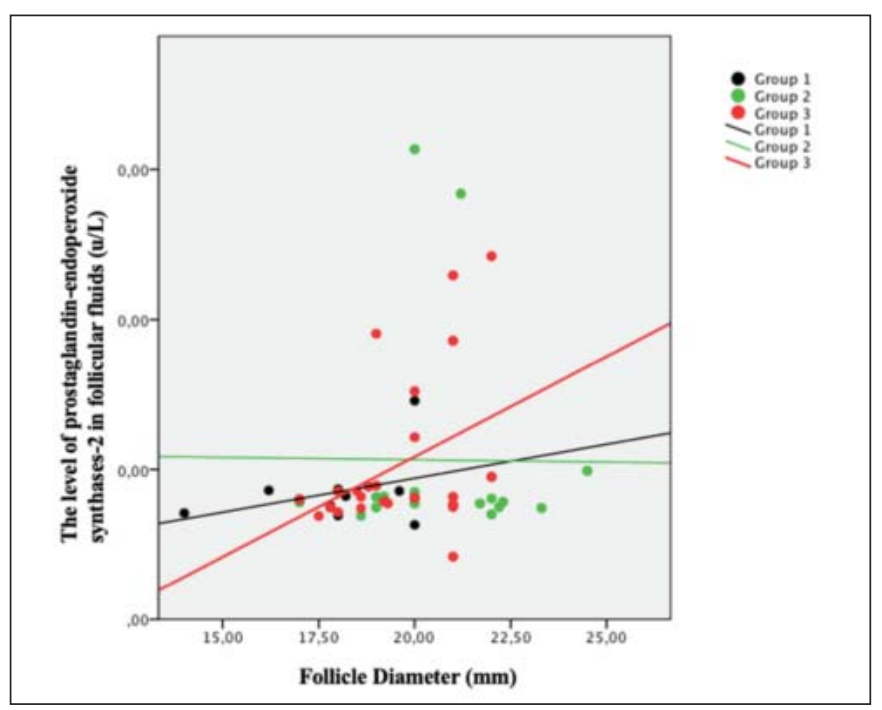

Figure 3: The correlations between follicle diameter and level of prostaglandin-endoperoxide synthases-2 (PTGS-2) in follicular fluids. With the level of PTGS-2 in follicular fluids levels only in Group 3, a statistically significant positive correlation was determined between follicular diameter and level of PTGS-2 in follicular fluid ( $r=0.414 ; p=0.035$ )

\section{Conclusion}

In our study, PTGS-2 gene expression levels in different nuclear maturation stages of human oocytes and in their surrounding CCs were evaluated additionally to FF PTGS-2 enzyme levels. Our results demonstrate that (i) FF PTGS-2 concentrations and follicle diameters showed a significantly positive correlation in oocytes that have completed their nuclear and cytoplasmic maturation, (ii) the relative PTGS-2 expression in CCs of immature oocytes was similar to that in mature oocytes CCs, and (iii) in terms of fertilization ability of oocytes, the expression of PTGS-2 in CCs of unfertilized oocytes were the same as in fertilized oocyte CCs.

Study samples were obtained from patients who underwent ovarian stimulation. In previous studies, it was suggested that controlled ovarian stimulation, compared to natural cycles, could cause changes in FF contents and this was also thought to affect CCs protein expressions (9). While this is the standard procedure for most research involving human

Table II: The mean follicular diameter in total and in groups

\begin{tabular}{|c|c|c|c|c|c|}
\hline & Total & Group $1(n=10)$ & Group 2 (n=16) & Group 3 (n=26) & $p$ \\
\hline Follicle diameter (mm) & $19.8(14-24.5)$ & $18(14-20) x$ & $20.6(17-24.5) y z$ & $19.65(17-22.5) z$ & $0.001^{*}$ \\
\hline $\begin{array}{l}\text { The level of prostaglandin-endoperoxide } \\
\text { synthases- } 2 \text { in follicular fluids (u/L) }\end{array}$ & $\begin{array}{c}201.94 \\
(83.96-626.88)\end{array}$ & $\begin{array}{c}162.18 \\
(126.17-291.58)\end{array}$ & $\begin{array}{c}156.51 \\
(138.31-626.88)\end{array}$ & $\begin{array}{c}163.63 \\
(83.96-484.83)\end{array}$ & 0.504 \\
\hline
\end{tabular}

The Kruskal-Wallis test for comparison of ranks between groups, data are given as median (min-max) 
oocytes and CCs, it must be stressed, though, that in order to minimize the influence of possible variations in the hormonal environment, this study was performed in patients with matching characteristics.

The important subject is the evaluation of the impact of follicle diameters on clinical results. The follicles with a smaller size (a diameter of 3-10 mm) were capable of containing mature oocytes with a decreased rate $(25.4 \%)$, while their fertilization rate was similar to that of MII oocytes obtained from larger size (a diameter of 16-18 mm) follicles (\%85.6, \%85.8 respectively) (15). Moreover, it was reported that fertilization rates evaluated for different follicle sizes as $<$ $15 \mathrm{~mm}, 15-18 \mathrm{~mm}$ and $>18.5 \mathrm{~mm}$ were $49 \%, 53 \%$ and $64 \%$ respectively in ICSI cycles. In the larger follicles, the rate of abnormal fertilization was also lower than in oocytes retrieved from smaller follicles (9\% vs 13\%, respectively) (16). In our study, contrary to previous findings, follicles that reached 20.6 (17-24.5) mm (Group 2) in diameter containing MII oocytes (Group 2) showed higher fertilization defects than mature oocytes obtained from follicles with a median diameter of 19.65 (17-22.5) mm (Group 3). It could be postulated that in mature oocytes obtained from larger follicles, the cutoff measurement with a gap of knowledge may be postmature - aged oocytes. Given that the evaluation of oocyte maturation is carried out according to nuclear maturation (excretion of pole polar body) and that the rate of cytoplasmic maturation can only be assessed by fertilization success, it can be concluded that the oocytes complementing the nuclear and cytoplasmic maturation do not correlate with the increase in follicle diameter, as supported by the work of Teramoto et al. (15).

Prostaglandin synthesis from ovarian follicles has also been reported in a previous study $(9,17,18)$. Several studies have reported that the expression of the PTGS-2 enzyme is responsible for prostaglandin E2 synthesis in CCs and their activity for CC expansion and oocyte maturation in rodents $(4,5,19,20)$. The increase in prostaglandin E2 levels for the in vitro maturation process, 6 hours, and before (i.e. before germinal vesicle destruction) (21) supports the idea that it is involved in oocyte maturation and cumulus expansion. Moreover, in the process of ovulation, hCG plus PTGS-2 inhibitor (celecoxib) application in a monkey model caused a decreased rate of mature oocytes, presumably due to the failure of both cumulus expansion and rupture of the follicle wall. Administration of high doses of the PTGS-2 inhibitor indomethacin to mice at the time of hCG administration also delayed oocyte nuclear maturation $(22,23)$. Administration of lower doses of indomethacin yielded primarily MII oocytes, even when follicle rupture failed (24), suggesting that follicle rupture is more sensitive to inhibition of PTGS-2 than is oocyte maturation. In our study, although a limited number of samples have been studied, relative PTGS-2 expression in CCs surrounding immature oocytes (Group 1) showed a tendency to increase compared to that of CCs surrounding mature, unfertilized oocytes (Group 2).
Conversely, it was observed that PTGS-2 expression in immature oocytes (Group 1) showed a greater tendency to decrease than in mature but not in fertilized oocytes (Group 2). Although with no significant difference, it could be speculated that the tendency of increased PTGS-2 gene expressions in immature oocytes and their CCs may lead to premature follicular rupture independently of the oocyte maturation stage. This suggestion could be demonstrated with a study that includes a larger patient sample.

This study was based on explaining a disrupted oocyte maturation process and fertilization defects with the gene expression of PTGS-2 in oocytes and surrounding cumulus cells and to analyze whether follicular diameter has a relation with the level of follicular fluid PTGS-2 in follicles that contain immature oocytes. To the best of our knowledge, the level of FF PTGS-2 concentration and the PTGS-2 expression in human oocytes and CCs were assessed for the first time according to the oocyte maturation level. There was found to be no significant efficacy of PTGS-2 gene expressions and levels in the oocyte maturation process and fertilization. However, the tendency of increased PTGS-2 gene expressions in immature oocytes and their CCs may lead to new clinical approaches in cases of premature follicular rupture or oocyte maturation arrest in-vitro. There is a need for more extensive research in which more material can be assessed and molecular mechanisms can be explored.

Acknowledgment: None

Compliance with ethical standards: Ethical approval was obtained for all procedures performed in the studies involving human participants, which were in accordance with the ethical standards of the institutional research committee and with the 1964 Helsinki Declaration and its later amendments.

Author's contributions: GK: Contributed to the conception and design of the study, review of the manuscript and approval of the final version. BA: Contributed to the conception and design of the study, review of the manuscript, and approval of the final version.

Funding: This study was funded by the Research Fund of the Bursa Uludag University School of Medicine, Bursa, Turkey. Conflict of interest: None of the authors have any conflict of interest associated with this study.

\section{References}

1. Wang D, Mann JR, Dubois RN. The role of prostaglandins and other eicosanoids in the gastrointestinal tract. Gastroenterology. 2005;128(5):1445-61. Doi:10.1053/j. gastro.2004.09.080.

2. Smith WL, DeWitt DL, Garavito RM. Cyclooxygenases: structural, cellular, and molecular biology. Annu Rev Biochem. 2000;69:145-82. Doi:10.1146/annurev.bioc hem.69.1.145.

3. Nuttinck F, Reinaud P, Tricoire H, Vigneron C, Peynot N, 
Mialot JP, et al. Cyclooxygenase-2 is expressed by cumulus cells during oocyte maturation in cattle. Mol Reprod Dev. 2002;61(1):93-101. Doi:10.1002/mrd.1135.

4. Lim H, Paria BC, Das SK, Dinchuk JE, Langenbach R, Trzaskos JM, et al. Multiple female reproductive failures in cyclooxygenase 2-deficient mice. Cell. 1997;91(2): 197-208. Doi:10.1016/s0092-8674(00)80402-x.

5. Hizaki H, Segi E, Sugimoto Y, Hirose M, Saji T, Ushikubi $\mathrm{F}$, et al. Abortive expansion of the cumulus and impaired fertility in mice lacking the prostaglandin E receptor subtype EP(2). Proc Natl Acad Sci U S A. 1999;96(18): 10501-6. Doi:10.1073/pnas.96.18.10501.

6. Matsumoto H, Ma W, Smalley W, Trzaskos J, Breyer RM, Dey SK. Diversification of cyclooxygenase-2-derived prostaglandins in ovulation and implantation. Biol Reprod. 2001;64(5):1557-65. Doi:10.1095/biolreprod64. 5.1557.

7. Ochsner SA, Russell DL, Day AJ, Breyer RM, Richards JS. Decreased expression of tumor necrosis factor stimulated gene 6 in cumulus cells of the cyclooxygenase- 2 and EP2 null mice. Endocrinology. 2003;144(3):1008-19. Doi:10.1210/en.2002-220435.

8. Sirois J, Sayasith K, Brown KA, Stock AE, Bouchard N, Doré M. Cyclooxygenase-2 and its role in ovulation: A 2004 account. Human Reproduction Update. 2004;10(5): 373-85. Doi:10.1093/humupd/dmh032.

9. Duffy DM. Novel contraceptive targets to inhibit ovulation: The prostaglandin E2 pathway. Hum Reprod Update. 2015;21(5):652-70. Doi:10.1093/humupd/dmv026.

10. Triwitayakorn A, Suwajanakorn S, Pruksananonda K, Sereepapong W, Ahnonkitpanit V. Correlation between human follicular diameter and oocyte outcomes in an ICSI program. J Assist Reprod Genet. 2003;20(4):143-7. Doi: 10.1023/a:1022977002954.

11. Wittmaack FM, Kreger DO, Blasco L, Tureck RW, Mastroianni L Jr, Lessey BA. Effect of follicular size on oocyte retrieval, fertilization, cleavage, and embryo quality in in vitro fertilization cycles: a 6-year data collection. Fertil Steril. 1994;62(6):1205-10. Doi:10.1016/s00150282(16)57186-6.

12. Ectors FJ, Vanderzwalmen P, Van Hoeck J, Nijs M, Verhaegen G, Delvigne A, et al. Relationship of human follicular diameter with oocyte fertilization and development after in-vitro fertilization or intracytoplasmic sperm injection. Hum Reprod. 1997;12(9):2002-5. Doi: 10.1093/ humrep/12.9.2002.

13. Dubey AK, Wang HA, Duffy P, Penzias AS. The correlation between follicular measurements, oocyte morphology, and fertilization rates in an in vitro fertilization program. Fertil Steril. 1995;64(4):787-90. Doi: 10.1016/s00 15-0282(16)57855-8.
14. Kasapoglu I, Kuspinar G, Saribal S, Turk P, Avcı B, Uncu G. Detrimental effects of endometriosis on oocyte morphology in intracytoplasmic sperm injection cycles: a retrospective cohort study. Gynecol Endocrinol. 2018;34 (3):206-11. Doi:10.1080/09513590.2017.1391203.

15. Teramoto S, Osada H, Sato Y, Shozu M. Nondominant small follicles are a promising source of mature oocytes in modified natural cycle in vitro fertilization and embryo transfer. Fertil Steril. 2016;106(1):113-8. Doi:10.1016/ j.fertnstert.2016.03.015.

16. Mehri S, Levi Setti PE, Greco K, Sakkas D, Martinez G, Patrizio P. Correlation between follicular diameters and flushing versus no flushing on oocyte maturity, fertilization rate and embryo quality. J Assist Reprod Genet. 2014;31(1):73-7. Doi: 10.1007/s10815-013-0124-9.

17. Narko K, Ritvos O, Ristimäki A. Induction of cyclooxygenase- 2 and prostaglandin F2 $\alpha$ receptor expression by interleukin-1 $\beta$ in cultured human granulosa-luteal cells. Endocrinology. 1997;138(9):3638-44. Doi:10.1210/endo. 138.9.5388.

18. Wissing ML, Kristensen SG, Andersen CY, Mikkelsen AL, Høst T, Borup R, et al. Identification of new ovulation-related genes in humans by comparing the transcriptome of granulosa cells before and after ovulation triggering in the same controlled ovarian stimulation cycle. Hum Reprod. 2014;29(5):997-1010. Doi:10.1093/humrep/ deu008.

19. Eppig JJ. Prostaglandin E2 stimulates cumulus expansion and hyaluronic acid synthesis by cumuli oophori isolated from mice. Biol Reprod. 1981;25(1):191-5. Doi:10.1095/ biolreprod25.1.191.

20. Takahashi T, Morrow JD, Wang H, Dey SK. Cyclooxygenase-2-derived prostaglandin E2 directs oocyte maturation by differentially influencing multiple signaling pathways. J Biol Chem. 2006;281(48):37117-29. Doi:10. 1074/jbc.M608202200.

21. Marei WF, Abayasekara DR, Wathes DC, Fouladi-Nashta AA. Role of PTGS2-generated PGE2 during gonadotrophin-induced bovine oocyte maturation and cumulus cell expansion. Reprod Biomed Online. 2014;28(3):388400. Doi: 10.1016/j.rbmo.2013.11.005.

22. Downs SM, Longo FJ. Effects of indomethacin on preovulatory follicles in immature, superovulated mice. Am J Anat. 1982;164(3):265-74. Doi:10.1002/aja.1001640307.

23. Downs SM, Longo FJ. Prostaglandins and preovulatory follicular maturation in mice. J Exp Zool. 1983;228(1):99108. Doi:10.1002/jez.1402280111.

24. Hayashi S, Noda Y, Matsumoto H, Mori T. Fertilizability of unovulated mature eggs following indomethacin administration in mice. Gamete Res. 1987;18(4):291-9. Doi:10.1002/mrd.1120180403. 